\title{
Longitudinal Profile and Effective Length of a Conventional Dipole Magnet
}

\author{
Jean-François OSTIGUY \\ Fermilab; P.O. Box 500, Batavia, IL 60510
}

\begin{abstract}
Magnetic saturation in the vicinity of longitudinal edges results in a small decrease in effective length with increasing excitation current. This phenomenon does not pose any problem in a machine where all dipoles are identical. In the Fermilab Main Injector lattice, dipoles of two different physical lengths are used. The effective length variation is caused by end effects and is essentially independent of the physical length of each type of magnet. However, the relative variation is larger for the short magnets. Since all dipole magnets are on a common bus, the closed orbit is modified in an energy dependent way. Although this effect can be compensated with corrector magnets, a better approach is to design the dipole magnets so as to minimize absolute effective length variations. This can be accomplished with a suitable longitudinal pole profile in the end region. Theoretical considerations and numerical calculations leading to the choice of this profile are presented.
\end{abstract}

\section{INTRODUCTION}

The net (horizontal) angular deflection experienced by a particle of momentum $p$ going through a dipole magnet is given by

$$
\theta=\frac{\Delta p}{p}=\frac{q}{p} \int_{-\infty}^{\infty} \beta_{z}(s) B_{y}(x, y, s) d s
$$

For highly relativistic particles, $\beta \simeq 1$. Accelerator magnets are generally designed in two dimensions. Since by symmetry, the field does not have any axial component in the midplane, a straightforward two-dimensional calculation tends to accurately predict the field in that plane. To scparate bulk saturation effects from the small changes in the longitudinal field profile, it is convenient to introduce an "effective" or "magnetic" length $L_{\varepsilon}$, defined as follows,

\footnotetext{
*Operated by the Universities Research Association, Inc., under contract with the U.S. Department of Energy.
}

with the origin of the coordinate system in the center of the magnet

$$
L_{e}(I)=\frac{1}{B_{y}(I ; 0,0,0)} \int_{-\infty}^{\infty} B_{y}(I ; 0,0, s) d s
$$

The deflection can then be written in the form

$$
\theta(I)=\frac{q}{p} B_{y}(I ; 0,0,0) L_{e}(I)
$$

When the permeability is infinite, the vertical field on axis and the effective length can be estimated by solving in the $y-s$ plane for the magnetic scalar potential assuming that the poles are equipotential surfaces. Unfortunately, this approach fails when the permeability is finite or when there is saturation; furthermore, it tends to overestimate the field beyond a distance of the order of one gap width away from the physical end of the magnet. A full threedimensional solution is required to correctly model nonuniform saturation and the faster field decay due to the finite width of the magnet.

It should not be too surprising that a simple rectangular end results in relatively large variations of $L_{e}$ with increasing current. The high flux density in the corner region causes non-uniform saturation that tends to make the flux in the end region weaker, resulting in a decreasing $L_{\varepsilon}$. Clearly, the poles must be profiled longitudinally to control nonuniform saturation effects. As a starting point, it is natural to consider two classical profiles: the Borda and the Rogowski profiles.

\section{Borda Profile}

The Borda profile (which has also been attributed to Helmholtz) is well-known in classical hydrodynamics. It corresponds to the the curve defined by the streamlines for a fluid issuing from a large vessel by an aperture in a plane wall. In magnetostatics, the streamlines correspond to lines of constant vector potential. When the permeability is infinite, the Borda profile is such that the magnitude of the magnetic flux density along the profile is constant. Since the saturation level depends on the magnitude of the field, it seems reasonable to expect that the Borda profile would help minimizing the variation in $L_{e}$ by making the 
end region saturate uniformly. We shall see that in practice, this is not really the case. An analytical expression for the Borda profile can be obtained using standard conformal mapping techniques [2]. In parametric form, the result is

$$
\begin{aligned}
& x=\frac{2 g}{\pi}\left\{\sin \psi-\log \tan \left(\frac{\psi}{2}+\frac{\pi}{4}\right)\right\} \\
& y=\frac{2 g}{\pi}\left\{\frac{2+\pi}{2}-(1-\cos \psi)\right\}
\end{aligned}
$$

where $0<\psi<\pi / 2$ and $2 g$ is the total gap in the uniform field region. The Borda profile is shown in figure 1 . Note that the ratio of the maximum to minimum aperture is $(\pi+2) / \pi$.

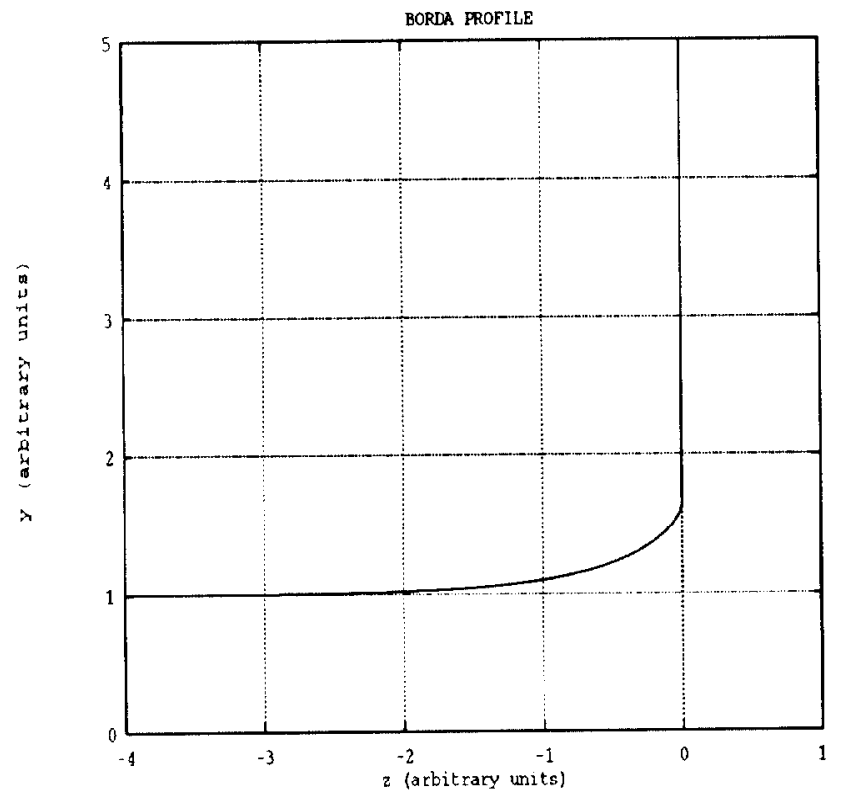

Figure 1: The Borda Profile.

\section{Rogowski Profile}

This profile was introduced in the context of study of electrical breakdown in gases. Rogowski realized that parallel metal plates of finite dimensions create a problem of electrical stress control at the edges of the plates. Depending upon the material to be tested, the breakdown strength may be very sensitive to local high fields within the whole electrode arrangement. Therefore, the highest stress should only be present in the homogeneous field region, where the plates are parallel. A certain profile of electrodes is necessary outside the plane region but the field strength at the curved edges should never exceed the value $H_{0}=V / 2 g$ where $V$ is the applied voltage and $2 g$ the distance between the plates. Rogowski proposed electrodes whose profile follows the analytical function

$$
z=\frac{2 g}{\pi}\left(w+1+e^{w}\right)
$$

or, with $z=x+i y$ and $w=u+i v$

$$
\begin{aligned}
& x=\frac{2 g}{\pi}\left(u+1+e^{u} \cos v\right) \\
& y=\frac{2 g}{\pi}\left(v+e^{u} \sin v\right)
\end{aligned}
$$

where $-\pi<v<\pi$ and $-\infty<u<\infty$. The function 6 was actually first introduced by Maxwell and describes the equipotentials lines of a semi-infinite capacitor. If the field in the uniform region is $H_{0}$, the magnitude of the field is

$$
|H|=\frac{H_{0}}{\sqrt{1+e^{2 u}+2 e^{u} \cos v}}
$$

When $-\pi / 2<v<\pi / 2$, the field strength has its maxi$m u m$ value in the homogeneous field region and decreases monotonically within the curvature with increasing values $u$. Rogowski chose the profile defined by $v=\pi / 2$ and $-\infty<u<\infty$ because it is the most compact profile that has this property. Since the field in the end region is always weaker than the body field, the end region can be expected not to saturate significantly until the body is well saturated. This should help keeping the effective length constant, at least for excitations below the level required to saturate the end region significantly. This approach turns out to work fairly well in practice. Note in passing that the Rogowski profile can also be defined as the profile which makes the vertical component of the flux density in the pole constant along the pole surface.

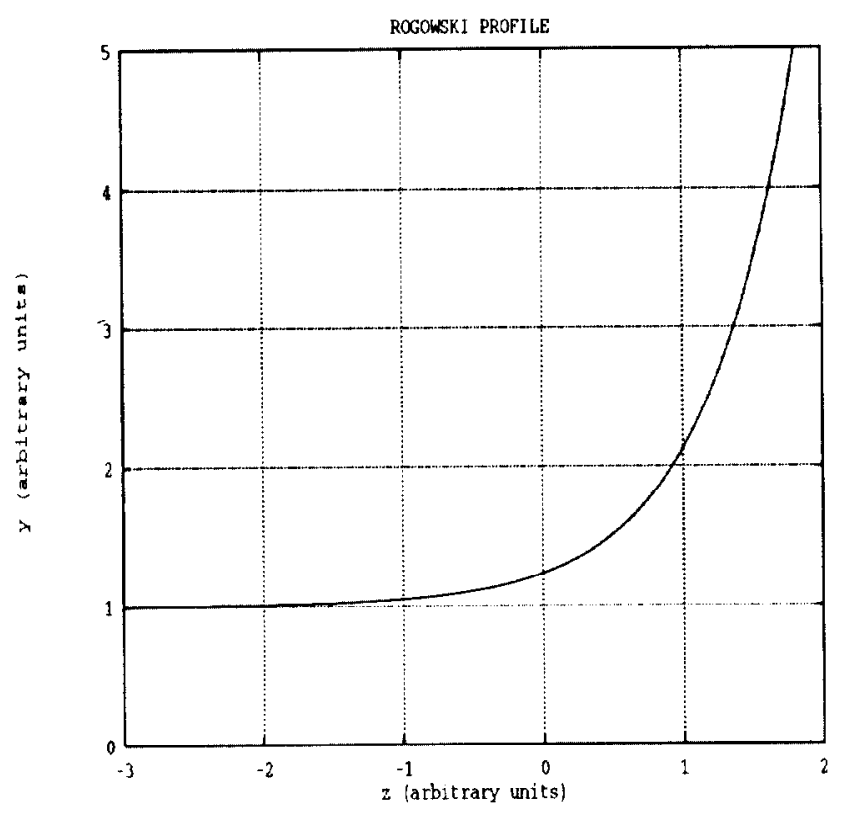

Figure 2: The Rogowski Profile.

The Rogowski profile is shown in figure 2 . 


\section{3D Calculations}

The initial attempts at computing changes in effective length for different profiles were not very successful. As part of the Main Injector magnet R\&D program, measurements were therefore performed on endpacks made out of stacked laminations, for both the Borda and Rogowski profiles [2]. The Borda endpack was measured first and the results were not encouraging; however, the Rogowski endpack met the specifications.

Better computer facilities subsequently became available and the calculations were performed again, this time with much improved resolution. The results for one end piece are presented in Table 1. Note that only the changes in length are significant. All calculations were made for one half of a magnet approximately $50 \mathrm{~cm}$ long. The agreement with the measurements presented in reference [2] is now relatively good, considering the fact that the computer model is an idealized smooth profile and that no attempt has been made to model the permeability anisotropy introduced by the laminations. For example, at 9500 Amperes, the model predicts a decrease of $6.6 \mathrm{~mm}$ per end for the Borda profile. The measured value is $4.5 \mathrm{~mm}$. For the Rogowski profile, the measured decrease is approximately $1 \mathrm{~mm}$ and the calculated value is $0.3 \mathrm{~mm}$.

\begin{tabular}{|l|l|l|l|}
\hline Profile type & $\mu=\infty$ & $I=7000 \mathrm{~A}$ & $I=9500 \mathrm{~A}$ \\
\hline \hline Borda Profile & $51.6612 \mathrm{~cm}$ & $51.6067 \mathrm{~cm}$ & $51.0017 \mathrm{~cm}$ \\
\hline Rogowski & $54.7420 \mathrm{~cm}$ & $54.7327 \mathrm{~cm}$ & $54.7116 \mathrm{~cm}$ \\
\hline
\end{tabular}

Table 1. Calculated Effective Lengths (3D Model)

The calculations also demonstrated that the problem with the Borda profile is the presence of rather non-uniform saturation in the bulk of the pole, right above the tapered region. The calculated longitudinal field profiles are illustrated in figure 3 . Note the almost linear decrease of the field within the body of the magnet.

\section{ACKNOWLEDGEMENTS}

The author would like to thank Dave Harding, Hank Glass and the personnel of the Fermilab Magnet Test Facility for their valuable comments.

\section{REFERENCES}

[1] J. Simkin and C. W. Trowbridge, "Three-dimensional Nonlinear Electromagnetic Field Computations, using Scalar Potentials", IEE Proc.,127,6, 368-374 (1980).

[2] D.J. Harding et al., "Design Considerations and Prototype Performance of the Fermilab Main Injector Dipole", These Proceedings.
[2] Horace Lamb, "Hydrodynamics", Cambdridge University Press 1879 (Reprinted by Dover Publications NY 1945).

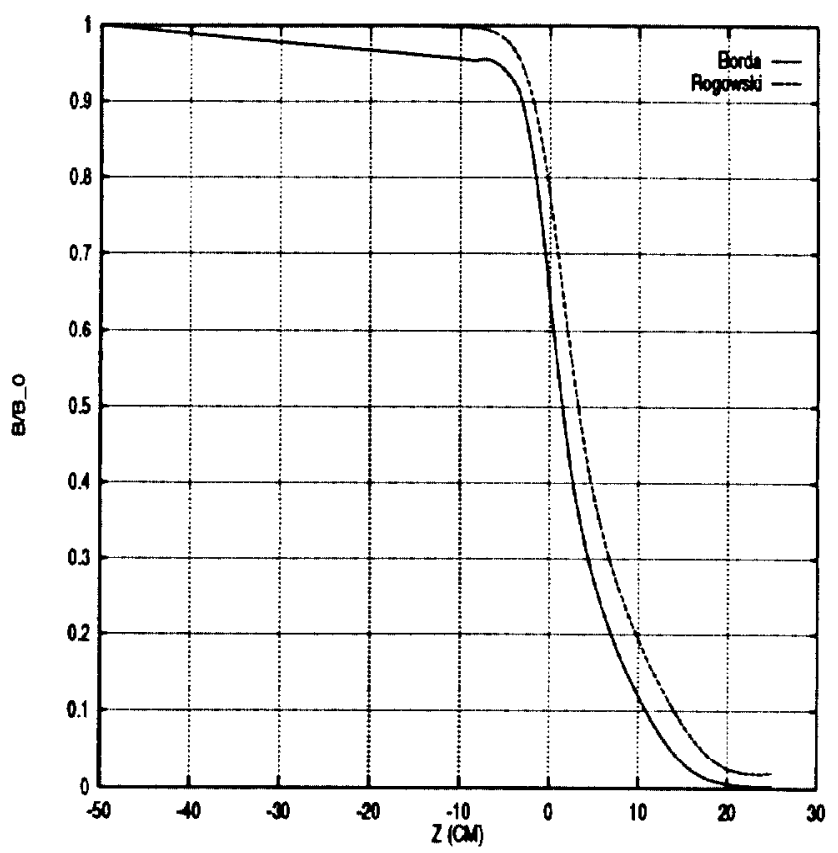

Figure 3: Calculated Longitudinal Field Profiles (3D model). 